\title{
Reflexiones sobre la calidad en la educación superior
}

\author{
Reflections on quality in higher education
}

\section{RESUMEN}

El artículo de reflexión aborda con metodologias del análisis hermenéutico, los conceptos claves de calidad educativa enmarcados en la tendencia global de un conocimiento pertinente. De igual forma se precisan las rutas para realizar un proceso de acreditación internacional asi como el rol de los docentes y los stakeholders que forman parte del constructo colectivo de la universidad y sus interrelaciones con la sociedad donde se desenvuelve.

\section{PALABRAS CLAVE}

Educación superior, Calidad educativa, Procesos de acreditación, Acreditacion internacional, Educación en el contexto global.

\section{ABSTRACT}

The article discusses with the methodologies of hermeneutic analysis, key concepts of educational quality framed in the global trend of relevant knowledge. Likewise, the routes to carry out an international accreditation process as well as the role of the teachers and the stakeholders that are part of the university's collective structure and its interrelations with the society where it is developed are also specified.

\section{KEYWORDS}

Higher education, Educational quality, Accreditation processes, International accreditation, Education in the global context.

\section{JUAN ALBERTO POLO FIGUEROA}

Doctor en Derecho y Ciencias Sociales de la Universidad Libre de Bogotá. Especialista en Administración Pública (Administración y Planificación del Desarrollo) de la Escuela Superior de Administración Pública de Bogotá. Se desempeñó como Magistrado del Consejo de Estado, siendo integrante de la Sala de lo Contencioso Administrativo, Sección Primera, en donde estuvo a cargo del conocimiento y decisión de controversias acerca de la constitucionalidad y legalidad de los actos jurídicos de la Administración Pública en todos sus niveles. Así mismo, fue Magistrado del Tribunal Administrativo del Magdalena y Decano de la Escuela de Derecho de la Universidad Sergio Arboleda Sede Santa Marta por más de una década. jopolofigueroa@gmail.com 


\section{INTRODUCCIÓN}

Asistimos en la actualidad a una nueva forma de entender los procesos; estamos justo en el momento en que pasamos de una cosmovisión lineal a una cosmovisión propia de lo incierto y caótico, es decir, abandonamos el paradigma de la modernidad cuya característica es lo predecible para adentrarnos a paradigma de lo postmoderno cuya esencia es lo impredecible. En esta ruptura o discontinuidad, entramos al fin de las certezas y necesitamos obligatoriamente refundar los criterios con los cuales se moldea la educación en este mundo. Manucci (2010, pp.11-12) lo expresa de la siguiente forma:

El final de las certezas al cual asistimos, en el inicio de una nueva década, no es más que el final de una cosmovisión imperante y hegemónica de ordenar e intervenir en la realidad. La pintura idílica de un mundo ordenado y previsible se ha transformado en una dinámica desbocada de consecuencias inciertas. ¿Qué está sucediendo nuestro alrededor? ¿Cómo llegamos a esta instancia? $\dot{ }$ El mundo ha estallado en múltiples procesos o el reloj que lo ordenaba dejó de funcionar? Probablemente estamos viviendo la misma incertidumbre

de aquel hombre medieval que miraba absorto su mundo cotidiano, que había dejado de ser una prolija trama de explicaciones aristotélicas y religiosas, para transformarse en un conjunto de incógnitas filosóficas, desafíos tecnológicos y amenazas espirituales. Aquellos hombres, en el límite del Medioevo, se enfrentaro a las paradojas de su momento histórico y fueron desarrollando explicaciones, teorías y métodos que dieron lugar a un nuevo ciclo de conocimiento y desarrollo. En este cambio de década, estamos recolectando las contradicciones y las paradojas que hemos construido con nuestras ideologías, metodologías y tecnologías que, en muchos aspectos, son extrapolaciones renovadas de aquella visión del mundo construida para reemplazar las explicaciones aristotélicas y religiosas de la realidad.

De ahí que en un mundo interconectado, las instituciones de educación superior no pueden permanecer al margen de los avances de saber en otras latitudes, ni rezagarse frente a los logros académicos alcanzados por otros establecimientos similares, sea dentro de su propio país o de otras naciones. La dinámica de la globalización presente en el mundo ha presionado a las universidades a una carrera competitiva, que hace imperativa la necesidad de poder cotejar el propio nivel de excelencia, a través de los procesos de evaluació y acreditación, a partir de estándares evidenciados de calidad comúnmente aceptados.

De esta labor de evaluación y acreditación de suma importancia, solo pueden ocuparse organismos que trasciendan lo local, idóneos, serios e imparciales, con la aptitud necesaria y reconocida para ser considerados evaluadores de la calidad de una determinada institución. Por iniciativa de la Asociación de Facultades, Escuelas e Institutos de Derech de América Latina, AFEIDAL, se ha constituido la agencia de acreditación internacional AlIDA, así mismo, la Asociación Colombiana de Facultades y Escuelas de Derecho, ACOFA$D E$, miembro activo de AFEIDAL, se ha comprometido con el buen suceso de la agencia de acreditación internacional, y al efecto asumió el cometido de conformar en Colombia un grupo de pares acreditadores internacionales.

En desarrollo de ese compromiso, en la ciudad de Santa Marta se realizaron dos sesiones de entrenamiento, dirigidas por expertos acreditadores mexicanos, quienes a través de sus exposiciones permitieron identificar los aspectos más relevantes imbricados en el concepto de la calidad en la educación superior, la importancia de la acreditación internacional, el proceso de auditoría o evaluación de las instituciones interesadas en obtener la acreditación, los indicadores a evalua y el papel y condiciones del par evaluador.

A los participantes en el seminario de formación, como culminación del proceso, se es impuso el deber de plasmar en un escrito las ideas y nociones aprehendidas, como un ercicio unificador de criterios. A ese deber concierne el presente trabajo, el cual se estructura sobre cuatro acápites principales: 1) el concepto de calidad en la educación superior, 2) las nociones que involucra, 3) la valuación y acreditación, y 4) los pares académicos.

\section{Qué significa calidad en la educación su-} perior?

Son múltiples los criterios que albergan el concepto de calidad en la educación superior, si bien parece evidente que se trata de que esta debe estar revestida de un alto grado de excelencia. La Asociación de Facultades, Escuelas e Institutos de Derecho de América Latina, AFEIDAL, ha propiciado múltiples certámenes académicos para discurrir acerca de la calidad en la educación superior; fruto de ello es la creación de una agencia internacional de acreditación de la alta calidad de las universidades de América, lo que supone tener definido el concepto de calidad y los parámetros para su medición.

En las memorias del 1er Congreso Internacional "La enseñanza del Derecho en América Latina y Bolivia" celebrado en la Universidad Santa Cruz de la Sierra, en mayo de 2004, el profesor Eugenio Hernández Aliste, Director de la Facultad de Ciencias Jurídicas de la Universidad Católica de Concepción, Chile, cuestiona: ¿Qué debemos entender por educación de calidad o calidad de la educación? y responde ofreciendo varias alternativas de respuesta:

a. Debemos entender por calidad de la educación el respeto, concreción y logro de la normativa, metas y objetivos planteados por la unidad académica o entidad de que se trata. En pocas palabras, se hace lo que se dice que se va a hacer. Aquí pondremos énfasis en el proceso, o sea, si se cumple lo que decimos que tenemos que 
hacer. Basta que se haga lo que se dijo que se iba a realizar. No existe preocupación por el contenido de la malla curricular, $\mathrm{ni}$ por la forma en que se entregan los conocimientos ni si los alumnos estarán en condiciones de desempeñarse adecuadamente en el ejercicio profesional.

b. Una segunda alternativa es la que dice relación con el entender por calidad de la educación el que en el proceso de enseñanza se vean involucrados profesionales de destacada trayectoria en sus respectivas áreas. $\mathrm{O}$ sea, se pretende relacionar con los pergaminos de los docentes, que en el resultado mismo del accionar educativo. No importa lo que el alumno aprenda, importa que quien enseña es e mejor. Pero el mejor en qué: en el ejercicio de su deber, pero esto está más que comprobado, NO ES GARANTÍA DE BUENA ENSEÑANZA. El enseñar implica no solo que yo sepa mucho, sino también el que yo logre que mis alumnos entiendan $y$ aprendan los conocimientos, desarrollen destrezas y capacidades que le permitan tilizar lo enseñado. Implica el tener que hacer entendible lo que para los alumnos aún no lo es.

c. La última alternativa y digamos que la indicaremos como la 'ultima' para estos fectos, es que entendamos por educación de calidad aquella que no descuida la malla curricular, asegurando contenidos mínimos; ni la enseñanza aprendizaje, asegurando con ello proceso; ni que los alumnos puedan aplicar sus conocimien- tos, o sea resultado del proceso" (Hernández Aliste, 2004).

De todo lo anterior Hernández Aliste (2004) concluye que:

Entenderemos por educación de calidad la que se preocupa de cada uno de estos aspectos: contenidos mínimos garantizados, procesos que aseguren adquisición de conocimientos y posibilidad cierta de aplicación en los cursos regulares de los conocimientos adquiridos.

A nuestro juicio, la calidad en la educación es mucho más que el currículo y los contenidos. Es una mera adquisición de conocimientos posibilidad de utilizar estos en los cursos regulares, siendo que la educación debe ser integral y para toda la vida. En ese sentido compartimos la idea del Ministerio de Educación Nacional de Colombia, MEN (2010), según el cual:

Educación de calidad es aquella que forma mejores seres humanos, ciudadanos con valores éticos, respetuosos de lo público, que ejercen los derechos humanos, cumplen con sus deberes y conviven en paz. Una educación que genera oportunidades legítimas de progreso y prosperidad. Una educación competitiva, pertinente, que contribuya a cerrar brechas de inequida $y$ en la que participe toda la sociedad. (p.12)

Son variados también los criterios bajo los cuales suele apreciarse el concepto de cali- dad en la educación superior, como el de la equidad, la eficiencia, y su relevancia. Para los fines de este artículo de reflexión, merecen una consideración especial los que se relacionan con la pertinencia, entendida como la necesidad de concebir una universidad eficaz, conveniente al entorno social; la internacionalización, referida al intercambio proesional, académico, cultural e investigativo en el ámbito de la competencia global; y e cumplimiento de estándares que responde a las exigencias académicas y sociales interinstitucionales. Es conveniente aclarar que cada uno de estos aspectos involucra a otros de trascendental significancia, sobre los cuaes se discurrirá a lo largo de este texto.

\section{2. ¿Cuáles son los conceptos claves}

Sobre la pertinencia. En primera instancia nos referiremos al criterio de pertinencia, definido por el pedagogo cubano, Águila Cabrera, como aquel que se mide por el impacto social que genera, por el flujo de repercusiones y de transformaciones de sentido que se producen objetivamente en la sociedad d su entorno. Así, es necesario educar para la pertinencia del conocimiento, de tal manera que este adquiera sentido en el contexto local, en consonancia con el ámbito global. Una educación de calidad debe aterrizar los conceptos abstractos en el contexto cotidiano. Los conocimientos adquieren valor cuando representan algo para la comunidad; esto es, cuando son útiles en la medida en que solucionan un problema, sin perder de vista que esa situación particular, local, está inmersa en un contexto global que es preciso considerar.
En este punto, es de crucial importancia la implementación de un enfoque curricular orientado no solo a la aprehensión del conocimiento, sino a su transferencia, dicho de otro modo, a la solución de problemas, de conflictos del entorno real, que le dan significado a la educación. En este propósito es determinante la enseñanza de la creatividad; al respecto Edgar Morin (2001) sostiene que:

(...) la educación debe favorecer a la aptitud natural de la mente para hacer y resolver preguntas esenciales $y$ correlativamente estimular el empleo total de la inteligencia general. Este empleo máximo, necesita el libre ejercicio de la facultad más expandida y más viva de la infancia y la adolescencia: la curiosidad (...). (p.14)

Educar para la pertinencia es preparar al individuo para el mundo local y global; es prepararlo para dar respuestas consecuentes, eficaces a los problemas sociales, y por ende, atender a las múltiples dimensiones del ser humano: la afectiva, cognitiva, biológica, psíquica, legal, estética, histórica, ambiental, entre otras tantas; unida a la capacidad de aprender y desaprender, condición tan necesaria en un mundo de constantes cambios.

En este orden de ideas, la universidad está llamada a preparar al hombre para la comprensión del mundo, para darle respuestas a los problemas del ser humano; de aquí la importancia de conocerlo, de conocernos. La pertinencia no debe ser sinónimo de utilitarismo, en el sentido de dar respuestas desprovistas de humanidad a las preocupacio- 
nes sociales. Las especializaciones, por ejemplo, deben apuntar al estudio minucioso de los infinitos aspectos del hombre y de su entorno, pero solo en función de su formación holística. La pertinencia obliga a las universidades a reconsiderar la formación integral del hombre para la comprensión del otro ser humano y de nuestro entorno, partiendo por supuesto, del estudio de las ciencias humanísticas.

Preparar al estudiante como un auténtico ser humano que interprete a los otros seres humanos, y presente soluciones armónicas desde el conocimiento y la experiencia, considerando su contexto local y global, provocando un impacto social, es educar en la pertinencia.

Sobre la internacionalización. Teniendo presentes las anteriores consideraciones, educar en la pertinencia, precisa, entonces, educar para el proceso de internacionalización. El hombre no puede dar respuesta a las situaciones de su contexto, desconociendo el proceso de comunicación e interdependencia económica, cultural, social, política de los países. La educación debe preparar al estudiante para afrontar exitosamente la competitividad que trae la globalización. El propósito de dotarlo de competencias académicas, investigativas, axiológicas, comunicativas, ciudadanas, y laborales, entre otras, debe conducirlo a entender, comprender $y$ responder a las necesidades de un contexto social local interrelacionado con otras sociedades, con el contexto global.
En este sentido, la educación universitaria debe estar encaminada a ofrecerle a los estudiantes, propuestas y planes que conlleven al conocimiento de otras sociedades $y$ culturas; los programas de inmersión e intercambio, para citar algunos, lo enfrentan a experiencias de vida para el conocimiento y comprensión de otra sociedad poseedora de toda una ideología y un estilo de vida que es preciso estudiar y considerar, con la intención de consolidar verdaderas alianzas que optimicen el nivel de vida.

Una de las competencias que facilitan aproximación a otra cultura es el desarrollo de la competencia comunicativa -lectura escritura- como eje transversal de todas las áreas del conocimiento, y aprehensión de entorno. Así, el dominio de una lengua extranjera, particularmente del inglés, como

la lengua utilizada en el ámbito comercia se constituye en una obligación. Sin embargo, es preciso recordar que en la mayoría de los estudiantes que acceden a la educación superior se evidencian conocimientos básicos -incipientes del inglés-, situación que puede estar asociada al enfoque pedagógico adoptado en los niveles precedentes -básico y medio-. Estos datos permiten recordar la obligación estatal y de las universidades en la viabilización del bilingüismo como una oportunidad de comunicación, y por ende, de preparación para la internacionalización que no es más sino el intercambio de experiencias en las comunidades del mundo.

Recapitulando, las condiciones críticas tanto económicas como ecológicas del sistema mundial, el auge de las relaciones internacionales, las comunicaciones en un mundo globalizado, las particularidades de Colombia y la realidad de una creciente brecha entre los países con sistemas económicos consolidados frente a países que padecen precariedades económicas, la condición de "Distrito Turístico" dado a nuestra ciudad de Santa Marta, requieren una redefinición del desarrollo humano y un nuevo énfasis sobre el conocimiento científico, humano y tecnológico en la educación superior. Este es un desafío que exige una nueva manera de percibir el mundo, de generar comunicación intercultural. Es preciso entonces, desarrollar las habilidades comunicativas necesaria para interactuar, y por tanto, el manejo del inglés es preponderante.

En Colombia, la Ley 115 determina, a parti del artículo 67 de la Constitución, como uno de los fines de la educación: "El estudio y la comprensión crítica de la cultura nacional $y$ de la diversidad étnica y cultural del país, como fundamento de la unidad nacional y de su identidad" (Título I, Artículo 6). Así mismo, el Ministerio de Educación Nacional de Colombia, MEN (1999) resalta la necesidad de promover la adquisición de por lo menos un engua extranjera desde el ciclo de primaria:

Se propende, entonces, porque la enseñanza de las lenguas extranjeras sea prospectiva para responder a las necesidades de la multiculturalidad. Corresponde esto a una visión prospectiva del siglo XXI en e que un alto porcentaje de los colombianos deberá tener acceso al conocimiento de las lenguas extranjeras como condición para mejorar el proceso de construcción de una sociedad más cohesionada, que presente las distintas identidades culturales con mayor equidad con un modelo de desarrollo sostenible personalizado. (p.15)

En esta misma vía, la internacionalización ha forzado a las universidades a la vinculación de las nuevas tecnologías de información y comunicación para optimizar la enseñanza y el aprendizaje, reducir la brecha digital y aprovechar las ventajas de la conectividad; asi mismo, en el entrenamiento en el uso de plataformas para entornos virtuales. Ahora resta que el docente asuma su verdadero compromiso con la educación superior en cuanto a su inclusión en las redes de investigación, que lo lleven a un verdadero intercambio del conocimiento, investigaciones y experiencias a nivel global, $y$ a la universidad a facilitar los recursos humanos, físicos y económicos. La internacionalización demanda la preparación integral de sus docentes, y por ende de sus estudiantes: ciencia, tecnología, investigación, comunicación con miras a la producción de nuevos conocimientos.

Se concluye este aparte, remarcando que la condición de apertura al contexto global que obliga a la entidad planetaria, no debe suponer la pérdida de identidad con el contexto local de nuestra tierra patria. El estudiante universitario debe educarse en el desarrollo de competencias que le permitan la comprensión y solución creativa de los problemas de su entorno primario, esto 
es, con la capacidad de aceptar al otro y su Por un lado, a considerar la existencia de los otra verdad, de atender a nuestra idiosincrasia, filosofía, economía, geografía, y otros múltiples factores que como país nos hacen diferentes, únicos; y que por tanto, exigen soluciones únicas, sin nunca perder de vista que somos parte de un gran contexto. Este pensamiento es el mismo que debe operar en las universidades colombianas, cada una es única por su razón académica y sociocultural expresada en su Misión, recreada en su currículo, condición que habla de su autonomía y libertad de cátedra; sin embargo como instituciones de educación superior convergemos en un mismo contexto que exige un trabajo mancomunado y armónico en pos de nuestra tierra patria, Colombia. En síntesis, la universidad debe enmarcarse en lo local con una perspectiva global, sin detrimento de su identidad.

\section{3. ¿Cómo se deben asumir los procesos de} evaluación y acreditación?

Hablar del concepto de calidad en la educación superior implica referirse imperativamente a los procesos de evaluación y acreditación. El proceso de internacionalización, como reacción a la globalización, ha representado la existencia de los sistemas de acreditación como forma de asegurar la calidad en la educación superior. En este sentido se han concebido los criterios o estándares como referentes de la calidad, entre una y otra universidad, enmarcada en el contexto nacional e internacional. Este enunciado conlleva a varias reflexiones: sistemas de acreditación como organismos de reconocimiento público y estímulo a los procesos realizados al interior de la universidad en consonancia con su contexto local global. La universidad debe mirar este proceso como una oportunidad para validar a nive nacional e internacional sus múltiples acciones desde las diferentes gestiones, dentro de las cuales impera la producción del conocimiento en bien de la humanidad.

Ahora bien, los sistemas de acreditación de ben convertirse en un ente dinamizador, oxigenador de la praxis académica, en cuanto verifique el cumplimiento de los estándare de calidad, y su sostenibilidad; por tanto la universidad debe estar vigilante a que estas prácticas no se conviertan en un mecanismo asfixiante, burocrático, mercantilista si se quiere, que degenere su propia razón de ser. En este propósito, el Estado debe asegurar la idoneidad de estos entes acreditadores.

Por otro lado, y teniendo como base lo expuesto anteriormente, se concibe la acreditación como una práctica que debe conducir principalmente a la cultura de la autoevaluación y la autorregulación porque la calidad es un proyecto inacabado. El diálogo entre pares académicos debe constituirse en un espacio para el debate bien intencionado, sobre los objetivos, acciones y proyectos de la universidad, en relación con las dimensiones de acción de la vida educativa de la universidad.

Los procesos de acreditación no deben ge- nerar una homogenización de la universidad por el contrario, deben garantizar la heterogenización, que no es más que el respeto por la autonomía universitaria y su propósito de pertinencia. Es la universidad la primera llamada en la búsqueda, el mejoramiento y el logro de la calidad a través de la autoevaluación y la autorregulación, procesos que requieren el compromiso y sentido de pertenencia de los entes principales de la comunidad universitaria.

En suma, la autoevaluación y autorregulación al interior de la universidad son procesos permanentes, sistemáticos y continuos que necesariamente conducirán a la acreditación; esta última debe constituirse en el efecto, no en la causa. Es claro que hasta ahora, los distintos países, casi que aisladamente, han venido impulsando procesos de acreditación que responden a su idiosincrasia y a sus propias circunstancias. A tal efecto han establecido lineamientos que permiten a sus universidades disponer de parámetros para la autoevaluación, permitiéndoles ajustar sus condiciones, mejorar sus condici y prepararse, frente a criterios claros y objetivos para superar el reto que entraña demostrar que la educación que imparten es de alta calidad.

. ¿Cuáles son los esquemas de un modelo de acreditación?

Bajo la consideración de que los procesos na- Como resultado de la visita puede establecionales guardan similitud con los de acreditación internacional que propicia AFEIDAL, se considera conveniente mostrar, de una par- te, el modelo seguido para el proceso mismo de acreditación en Colombia, y de otra, los estándares de calidad que se asumen ya que es a través de estos como se demuestra que se satisfacen criterios aceptables en la calidad de la educación, y se pueden realizar cotejos, equivalencias e intercambios de información y resultados.

A. Condiciones iniciales para la acreditación: Con el propósito de que las universidades o instituciones estén conscientes de si se encuentran en condiciones de adelantar, con probabilidades de éxito, un proceso de acreditación, el Consejo Nacional de Educación Superior ha establecido un procedimiento encaminado a la iniciación del proceso de autoevaluación con miras a la acreditación.

Estas condiciones iniciales las verifica el Consejo Nacional de Acreditación, plasmadas en el Acuerdo 02 del 18 de abril de 2012, el cual verifica la existencia de sujeción de la Institución a la normatividad legal, la existencia de una Visión que la defina institucionalmente, de un proyecto educativo, de un núcleo de profesores de planta, estatutos y reglamentos de docentes y estudiantes, de productividad académica de los profesores, de una estructura organizacional administrativa y de gestión, y finalmente, de una infraestructu-

ra locativa y logística (recursos académicos), para mantener el bienestar de sus miembros. cerse que la Institución cumple con las condiciones requeridas, en cuyo caso podrá iniciar el proceso de acreditación, o de no cumplir- 
las, se le permitirá solicitar una nueva visita de apreciación de condiciones iniciales.

B. Autoevaluación interna: Consiste en un ejercicio permanente, participativo, de revisión y de regulación realizado al interior de la Institución para verificar el grado de desarrollo de las funciones sustantivas de la universidad.

C. Autoevaluación externa: A cargo de pares académicos, que permiten formar un juicio sobre la calidad del Programa.

D. Evaluación final por el Consejo Nacional de Acreditación.

\section{Conclusión: los factores de calidad y el rol} del par

La evaluación que realiza el Consejo Naciona de Acreditación gira alrededor de diez factores, que integran el modelo de acreditación Estos factores son:

1. Misión, Visión y Proyecto Institucional y del Programa

2. Estudiantes

3. Profesores

4. Procesos académicos

5. Investigación y creación artística y cultu-

6. Visibilidad nacional e internacional

7. Impacto de los egresados sobre el medio

8. Bienestar Institucional

9. Organización, administración y gestión

10. Recursos físicos y financieros.
Cada uno de estos factores se compone de varias características y la identificación de los aspectos a evaluar. La identificación de indi-

cadores para demostrar el cabal desarrollo

de los factores ha sido suprimida por el Con-

sejo Nacional de Acreditación. En contraste,

la Agencia Internacional de Acreditación

AIDA, aplica indicadores como fórmula de evaluación, los cuales pueden ser conocidos previamente por las instituciones o universdades que aspiren a ser acreditadas internacionalmente.

Los procesos de acreditación internaciona serán exitosos en la medida en que las agencias correspondientes actúen con absoluta seriedad, con máximo rigor académico y sin cosiones de ninguna índole.

Papel importante les corresponde a los pares académicos, quienes deben ser conscientes de su trascendente misión. Son pares, es decir, iguales a los directivos y docentes quienes van a evaluar, mas están revestidos de una facultad especial que les permite adoptar decisiones en las cuales juegan su prestigio y el respeto que merecen. El par académico internacional no puede ser un fiscal sino un acompañante en el proceso de calidad de la Institución que se le confía, comprometido con sus éxitos.

Creemos que en la formación de pares académicos internacionales, AFEIDAL ha venido dando certeros pasos y que en breve se apreciarán sus frutos.

\section{REFERENCIAS BIBLIOGRÁFICAS}

Hernández Aliste, E. (2004). ¿Qué debemos entender por educa dad de la educación? En F. E. Núñez Jiménez (Ed.), La enseñanza del Derecho en América Latina y Bolivia (pp.54-70). Universidad Privada de Santa Cruz de la Sierra, Instituto de Investigación Jurídica, Facultad de Ciencias Jurídicas y Sociales, Bolivia. Hernández Aliste E. (2004). Memoria del I la prosperidad. Santafé de Bogotá D.C.: MaCongreso Internacional: Enseñanza del Dere- gisterio.

cho en Latinoamérica. Universidad de Santa

Cruz de la Sierra. Ley 115 (8 febrero de 1994). Por la cual se Unesco, Magisterio.

expide la Ley General de Educación. bia, MEN (2010). Educación de calidad para

Morin, E. (2001). Los siete saberes necesarios para la educación del futuro. Bogotá:

Manucci, M. (2010). Contingencias. Bogotá: Grupo Editorial Norma.

Ministerio de Educación Nacional de Colompara ldiomas Extranjeros. Áreas Obligatorias Fundamentales. Santafé de Bogotá D.C.: 